\title{
Concepción de Estudiantes y Docentes del Buen Profesor Universitario. Facultad de Medicina de la Universidad de La Frontera
}

\author{
Thinking of Students and Teachers of Good University Teacher. \\ Faculty of Medicine Universidad de La Frontera
}

"Daisy Cabalín Silva; **Nancy Navarro Hernández; ${ }^{* * * *}$ José Zamora Silva \& ${ }^{* * * * *}$ Silvia San Martín González

CABAlín, S. D.; NAVARRO, H. N.; ZAMORA, S. J. \& SAN MARTíN, G. S. Concepción de estudiantes y docentes del buen profesor universitario. Facultad de medicina de la universidad de la frontera. Int. J. Morphol., 28(1):283-290, 2010.

RESUMEN: Actualmente la calidad del Profesor Universitario pasa por los nuevos roles que debe asumir acorde al paradigma educativo de centrar el proceso enseñanza-aprendizaje en el estudiante. Es por ello que el presente estudio busca explorar la representación que estudiantes y docentes de la Facultad de Medicina de la Universidad de La Frontera tienen en relación al concepto "Buen Profesor Universitario". El estudio se enmarca en una investigación cualitativa permitiendo acceder al mundo conceptual de los individuos y a las redes de significados utilizando la técnica de Redes Semánticas Naturales. Se aplicó la encuesta a 104 docentes de diferentes profesiones y 293 estudiantes del último año curricular. Cada uno de ellos expresó con diez palabras la representación del concepto y la jerarquización según la importancia que ellos le asignaron. A los participantes del estudio se les solicitó su consentimiento, garantizando el anonimato y la confidencialidad de los datos. Emergieron de los docentes 228 palabras definidoras del concepto y de los estudiantes 248 . Los docentes dan mayor peso semántico a "Responsabilidad" (100\%), "Empatía" (97,6\%) y "Conocimiento" (60,3\%). Y los estudiantes priorizaron la "Responsable" (100\%), "Respetuoso" (89,4\%) y "Empático" (71\%) como principales atributos del Buen Profesor Universitario. En segundo nivel los docentes destacan aspectos relacionados con la cognición y los saberes, conocimiento 60,3\%, comprometido 49,4\%, actualizado 48,4 , estudioso $43,3 \%$ y los estudiantes con los aspectos de inteligente $47 \%$, puntual $42,7 \%$, compromiso $37,9 \%$ y motivador $36 \%$. Se destaca la importancia que tanto para docente como estudiantes tiene la responsabilidad y la empatía relacionadas con competencias del saber ser y saber convivir. Para los académicos la segunda área de importancia lo constituyen las competencias del saber valorando con ello la formación continua, a diferencia de los estudiantes para quienes este aspecto no tiene mayor significación.

PALABRAS CLAVE: Perfil profesor universitario; Redes semánticas; Competencias.

\section{INTRODUCCIÓN}

Lo vertiginoso del crecimiento del conocimiento, la falta de respuestas en los modelos conductistas para dar cuenta del como enseñar y aprender, los requerimientos de calidad que se exige a la tarea universitaria, hacen que cada día se cuestione con mayor rigurosidad a los actores que participan en la formación de los futuros profesionales que requiere un país. Sea cual fuere el número de factores o el peso con que cada uno de ellos influye en el logro de los aprendizajes requeridos para cualquier profesional, seguirá siendo en los diferentes modelos educativos, la calidad del profesor, que deberá desarrollar el proceso educativo, el responsable fundamental de la calidad del mismo.

Ya a principios del siglo pasado Dewey (1995) no sólo anticipó las líneas de pensamiento educativo sino que sus ideas también apuntaban al importante rol que tiene el profesor tanto en la entrega de conocimientos y habilidades como en su función formadora ética, valórica y de buena ciudadanía, lo cual sigue teniendo fuerza hasta hoy. Dewey, 1995 da una importancia fundamental a la figura del docen-

\footnotetext{
Profesora, Magíster Pedagogía y Gestión Universitaria. Oficina de Educación en Ciencias de la Salud (OFECS). Fac. Medicina, Universidad de La Frontera, Chile. ** Matrona, Magíster Pedagogía y Gestión Universitaria. Oficina de Educación en Ciencias de la Salud (OFECS). Fac. Medicina, Universidad de La Frontera, Chile.

*** Tecnólogo Médico, Magíster Pedagogía y Gestión Universitaria. Oficina de Educación en Ciencias de la Salud (OFECS). Fac. Medicina, Universidad de La Frontera, Chile.

***** Nutricionista, Magíster Pedagogía y Gestión Universitaria. Magíster en Evaluación. Oficina de Educación en Ciencias de la Salud (OFECS). Fac. Medicina, Universidad de La Frontera, Chile.
} 
te como educador, quién debe ver al estudiante como una persona en toda su integridad, ante todo, la función del ser docente, radica en la sabiduría como persona, con valores éticos que apunten al respeto a la dignidad del ser humano, que se dirijan a la formación del ciudadano democrático, demostrando con su ejemplo, lo que se espera de él.

Actualmente la calidad del Profesor Universitario pasa por los nuevos roles que le exigen los procesos educativos que apuntan a centrar el proceso en el aprendizaje y en el estudiante. Adicionalmente, la necesidad de incorporar competencias genéricas en la formación de los futuros profesionales (Tuning Educational Structures in Europe, 2003), las características evidenciadas y revaloradas hoy de cómo aprenden los adultos (Knowles, 1975), y el fortalecimiento de los cuatro pilares de aprendizaje referidos en el Informe Delors (1996), hacen inevitable una profunda renovación del como abordar la docencia universitaria y el rol que el docente debe asumir ante los nuevos desafíos.

El estudio de las características de un buen profesor universitario ha sido abordado por varios autores, Santos (1990) señala que debe dominar además de los saberes propios de su especialidad, otros de carácter pedagógicos, ha de saber que sucede en el aula, como aprenden los alumnos, cómo se puede organizar para ellos el espacio y el tiempo, que estrategias de intervención utilizar, también ser especialista en el diseño, desarrollo, análisis y evaluación de su propia práctica. Torres et al. (2007) concluye en su trabajo que dentro de las principales características del buen profesor universitario identificado por los académicos corresponden a: la actualización en su materia, poseer conocimientos de la materia que imparte, ser creativo, ser responsable, al dominio de la pedagogía y facilidad de palabras. Por otra parte, Manso (2005) menciona que debe ser un gestor de las actividades de aprendizaje, un generador de actitudes, un modelo profesional y humano de imitar, que conciba el aprendizaje como algo emocional no sólo cognitivo, con formación psicopedagógica e investigar sobre su propia docencia.

Otros como Arbizu (1994) consideran adjetivos como, especialista en diagnóstico, facilitador del aprendizaje, promotor de relaciones humanas, orientador, promotor, de par, de recurso, entre otros. Sin embargo, la apreciación de un "buen profesor universitario" esta generalmente planteado desde la perspectiva de los pares universitarios quienes tienden a asociarlo con que éste sea un buen investigador (Ballantine, 1989; Clark, 1986).

Por otro lado, la valoración de buen profesor universitario desde la perspectiva de los estudiantes, ha sido estudiada en alumnos de primer año de carreras de la salud por Cabalín \& Navarro (2008) quien señala que la conceptuali- zación que estos tienen del "buen profesor universitario", en primer lugar están relacionados con atributos valóricos (respetuoso, responsable), características personales que favorecen las interrelaciones que se establecen entre docentes y estudiantes (comprensivo, empático) y atributos propios para enfrentar la práctica educativa (claro, organizado, motivador). Corona (2008), indagando en estudiantes de ciencias físico-matemáticas encontró que las principales características del buen maestro están en el área académica (conocimientos, prepara su clase) en lo didáctico (explique bien) y en lo social (amable y puntual).

En un estudio con dos mil estudiantes Universitarios de Polonia, Nowakowski (2007) mencionan cualidades destacables en el área pedagógica (profesionalismo, objetivo, motivador) y en la perspectiva ética (respeto y amabilidad). Cataldi \& Lage (2004), encuestando a 200 universitarios de la Facultad de Ingeniería de la Universidad de Buenos Aires, concluye que ellos fundamentan sus necesidades desde un aspecto social, solicitando sobre todo buen trato, y que se interesen por ellos. Asimismo Verona (2004) haciendo una reflexión sobre la Universidad y el profesor universitario, después de una revisión de diversos autores concluye que existen diferencias entre como valoran los alumnos en el mundo anglosajón y el francófono, así los anglosajones destacan dos áreas, primero las que se centran en los valores de la personalidad y afectividad del profesor (estimulante, motivador) y una segunda las que se concentran en competencias intelectuales y pedagógicas del profesor. En cambio los francófonos dan más importancia a las cualidades pedagógicas y después aparecen características como respeto por los estudiantes, gusto por enseñar, disponibilidad, etc.

La Facultad de Medicina de la Universidad de La Frontera, inició a partir del año 2003 una innovación educativa centrando este proceso en el estudiante, por ello el presente trabajo busca indagar acerca de la representación que estudiantes y profesores construyen a partir del uso de redes semánticas sobre el concepto "Buen profesor Universitario", observar si hay coincidencias propias de una construcción socio-cultural común o discrepancias dados los roles dicotómicos que juegan estos actores.

\section{MATERIAL Y MÉTODO}

Dado el objetivo del estudio, éste se enmarca en una investigación cualitativa, permitiendo acceder al mundo conceptual de los individuos y a las redes de significados que tienen estudiantes y docentes en relación al concepto "Buen Profesor Universitario". 
Según Taylor \& Bogdan (1986) este enfoque investigativo produce datos descriptivos, utilizando las propias palabras de las personas "habladas o escritas", se realiza en escenarios naturales, estudiando a las personas en su propio marco de referencia. Para ello, se utilizó la técnica de redes semánticas naturales, como un conjunto de conceptos elegido por la memoria a través de un proceso reconstructivo que se desarrolla para explicar la forma en que se organiza la información frente a los conceptos que las personas tienen de cualquier objeto de su entorno, aproximando el significado de manera natural, es decir directamente con los individuos involucrados en el estudio (Figueroa et al., 1981; Chomsky, 1971).

La población del estudio estuvo constituida por estudiantes de las carreras de la salud que cursaban el último año curricular, antes de comenzar la etapa de las Prácticas Profesionales y docentes adscritos a los diferentes departamentos agrupados por profesión de la Facultad de Medicina de la Universidad de La Frontera. La muestra de los estudiantes estuvo conformada por 293 estudiantes correspondiendo a: Enfermería (44), Kinesiología (48), Medicina (37), Nutrición (30), Obstetricia (25), Odontología (75) y Tecnología Médica (34). Se consideró para el estamento docente encuestar a una muestra aproximada de un 10\% de la planta académica de la Facultad, participando en total 104 profesores: Enfermería (14), Kinesiología (15), Medicina (19), Nutrición (12), Obstetricia (13), Odontología (12) y Tecnología Médica (19). Tanto estudiantes como docentes fueron invitados a participar, informándoles sobre el propósito del estudio, solicitando su consentimiento, garantizando el anonimato y la confidencialidad de los datos.

Se aplicó la técnica de redes semánticas naturales a ambos grupos solicitándoles que frente a la palabra estímulo "Buen Profesor Universitario", expresaran con diez pala- bras la representación del concepto, luego las jerarquizaran de acuerdo a la importancia asignada por ellos, siendo el número uno la palabra mejor definidora y el número diez la más lejana al concepto, en un tiempo de cinco minutos la primera etapa y tres minutos la segunda.

Para el análisis de las redes semánticas naturales se consideraron los indicadores fundamentales descritos por Figueroa et al., Lagunas (1993), Valdez (1998), Valdez \& Reyes (1992): tamaño de la red (TR) "valor J" (total de las palabras definidoras para definir la palabra estímulo), peso semántico "valor M" (valor obtenido de la multiplicación entre la frecuencia de aparición por la jerarquía obtenida), conjunto SAM (grupo de las palabras que obtienen los mayores "valores M") y valor FMG (palabras definidoras del conjunto SAM, en términos de porcentajes, de la distancia semántica entre palabras tomando la palabra con mayor peso semántico como referencia del 100\%). El criterio de credibilidad del estudio se garantizó mediante la triangulación de los investigadores y la evidencia de los procedimientos realizados

\section{RESULTADOS}

De los 104 profesores participantes en el estudio de la Facultad de Medicina de la Universidad de La Frontera, la Figura 1 muestra la distribución de ellos por carrera en forma porcentual. De los docentes emergieron 228 palabras definidoras para la palabra estímulo "Buen Profesor Universitario", con un promedio de ocho palabras por encuesta. El resultado del conjunto SAM para las doce palabras con mayor peso semántico (Valor M) se representan en la Tabla I y en la Figura 2 que muestra la representación de los núcleos semánticos.

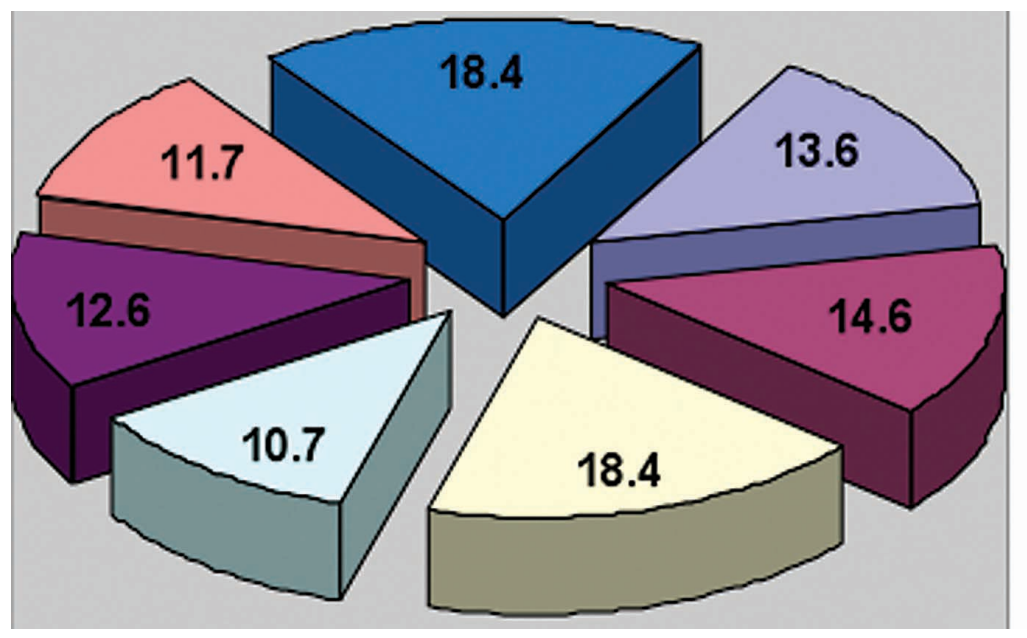

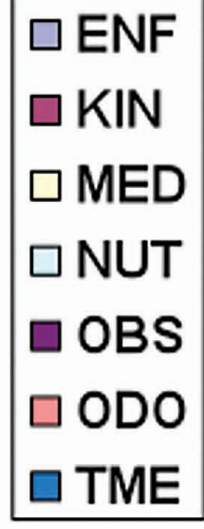

Fig. 1. Distribución Docentes por Carrera. 
Tabla I. Conjunto SAM de los profesores sobre el concepto "Buen Profesor Universitario".

\begin{tabular}{llcc}
\hline $\boldsymbol{N}^{\boldsymbol{o}}$ & \multicolumn{1}{c}{ Palabra Definidora } & $\begin{array}{c}\text { Peso Semántico } \\
\text { Valor } \boldsymbol{M}\end{array}$ & $\begin{array}{c}\text { Valor FMG } \\
\text { \% }\end{array}$ \\
\hline 1 & Responsable & 378 & 100 \\
2 & Empático & 369 & 97,6 \\
3 & Conocimiento & 228 & 60,3 \\
4 & Comprometido & 187 & 49,4 \\
5 & Actualizado & 183 & 48,4 \\
6 & Estudioso & 164 & 43,3 \\
7 & Ético & 150 & 39,6 \\
8 & Capacitado & 146 & 38,6 \\
9 & Honesto & 129 & 34,1 \\
10 & Respetuoso & 125 & 33,1 \\
11 & Inteligente & 103 & 27,2 \\
12 & Proactivo & 89 & 23,5 \\
\hline
\end{tabular}

\section{Valor FMG}

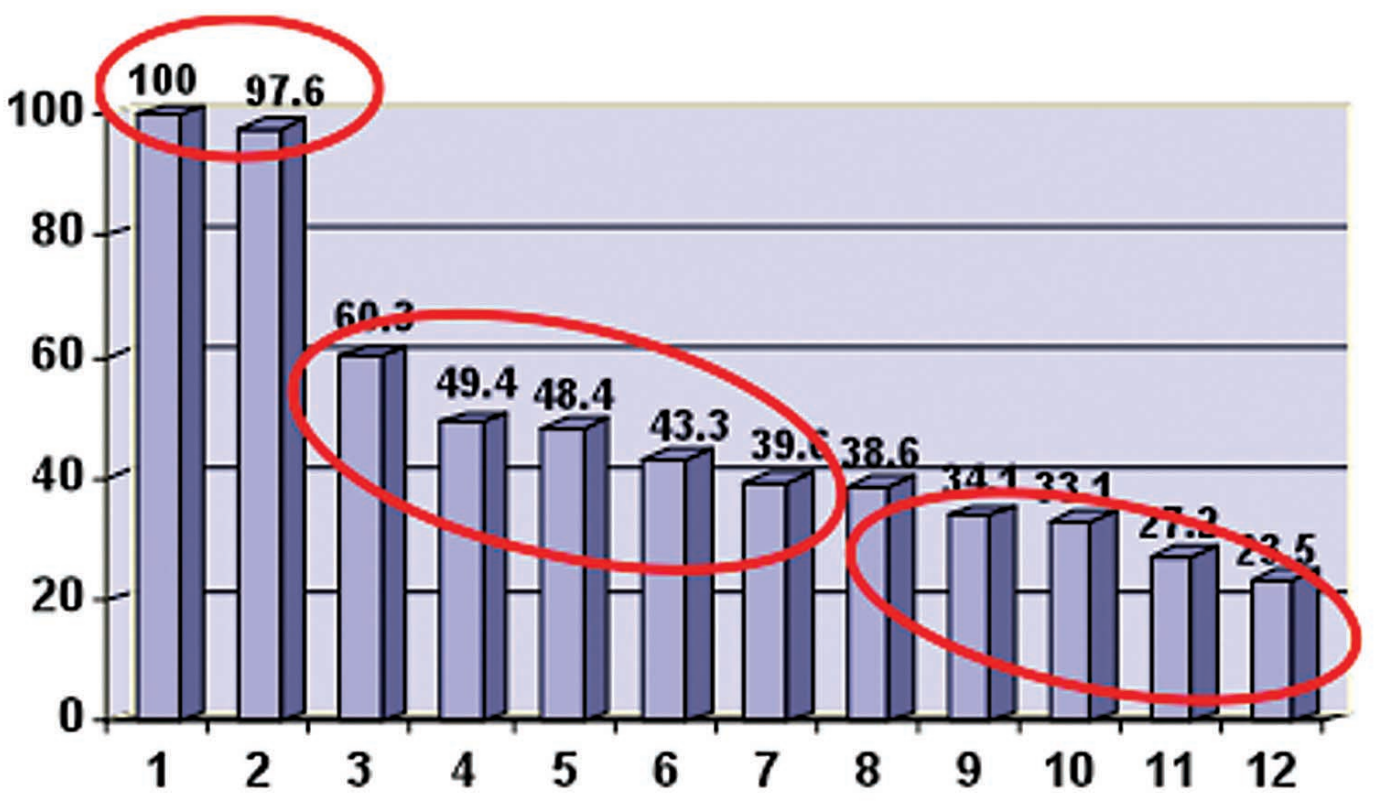

Fig. 2. Representación de los núcleos semánticos de los profesores del conjunto SAM.

En este contexto, el primer núcleo de palabras más relevantes como rasgos esenciales que fueron identificados son la "Responsabilidad" (100\%) y la "Empatía" (96,1\%), el primero tiene relación con cumplimiento de la función de educador y el segundo con ponerse en el lugar del otro. El segundo núcleo de palabras Conocimiento (76\%), Estudioso (69,6\%), Actualizado (58,3\%), Capacitado (55,4\%), Comprometido $(50,5 \%)$, están relacionados con la parte cognitiva, de saberes, expertizaje y dominio de la materia que el docente enseña. Además, valoran la formación continúa y el compromiso con la tarea docente. En el tercer núcleo temático se encuentran los conceptos de Honesto (44,1\%), Respetuoso $(43,6 \%)$, Proactivo (33\%), que hacen relación a características actitudinales del docente. Otros dos conceptos que se encuentran en este núcleo son Competente (30,9\%) e Inteligente $(28,4 \%)$ relacionados con las condiciones personales. 
Los 293 estudiantes que participaron en el estudio de todas las Carreras de la Facultad de Medicina corresponden a los últimos niveles de su formación previa a su Práctica Profesional. La Tabla II muestra el total de estudiantes por carrera, sexo y nivel curricular. Ellos emitieron 248 palabras definidoras frente al concepto "Buen Profesor Universitario" con un promedio de 9 palabras por encuesta. A continuación la Tabla III muestra el conjunto SAM para las doce palabras que representan las mayores frecuencias y la Figura 3 la representación de los núcleos semánticos.

Para los estudiantes el primer núcleo de palabras las constituyen "Responsable" (100\%), "Respetuoso" (89,4\%) y "Empático" (71\%). Sólo estas tres palabras agrupan más del $50 \%$ de todas las opiniones de los estudiantes, es decir, el área de mayor interés para ellos. El segundo núcleo esta constituida por los conceptos "Inteligente" (47\%), "Puntual" (42,7\%), "Compromiso" (37,9\%) y "Motivador" (36\%), que tienen relación con características personales y actitudes. El tercer núcleo lo conforman las palabras "Conocimiento" (27,1\%), "Amable" (25.6\%), "Actualizado" (24,7\%), "Comprometido" (23\%), "Exigente" (20,2\%), relacionados con la formación continua del docente, con su actitud como profesor y en su relación con otros.

La Tabla IV muestra el total de palabras definidoras del concepto en estudio por Carrera y las tres palabras con mayor porcentaje, con el fin de señalar similitudes y diferencias. Para la mayoría las dos palabras más representativas son "Responsable" y "Respetuoso" y como tercera palabra aparecen "Empático", "Inteligente", "Comprometido", "Comprensivo", "Conocimiento y "Puntual".

Tabla II. Características del estudiantado.

\begin{tabular}{lccccc}
\hline \multicolumn{1}{c}{ Carrera } & Nivel & \multicolumn{2}{c}{ Estudiantes } & Mujeres & Hombres \\
& & $\boldsymbol{N}^{\boldsymbol{0}}$ & $\boldsymbol{\%}$ & & 19 \\
\hline Kinesiología & 8 & 48 & 16,4 & 29 & 6 \\
Enfe rmería & 8 & 44 & 15,0 & 38 & 25 \\
Medicina & 10 & 37 & 12,7 & 12 & 3 \\
Obstetricia & 8 & 25 & 8,5 & 22 & 1 \\
Nutrición & 8 & 30 & 10,2 & 29 & 21 \\
Tecnología Médica & 8 & 34 & 11,6 & 13 & 40 \\
Odontología & 10 & 75 & 25,6 & 35 & $\mathbf{1 1 5}$ \\
Total & & $\mathbf{2 9 3}$ & $\mathbf{1 0 0}$ & $\mathbf{1 7 8}$ & \\
\hline
\end{tabular}

\section{Valor FMG}

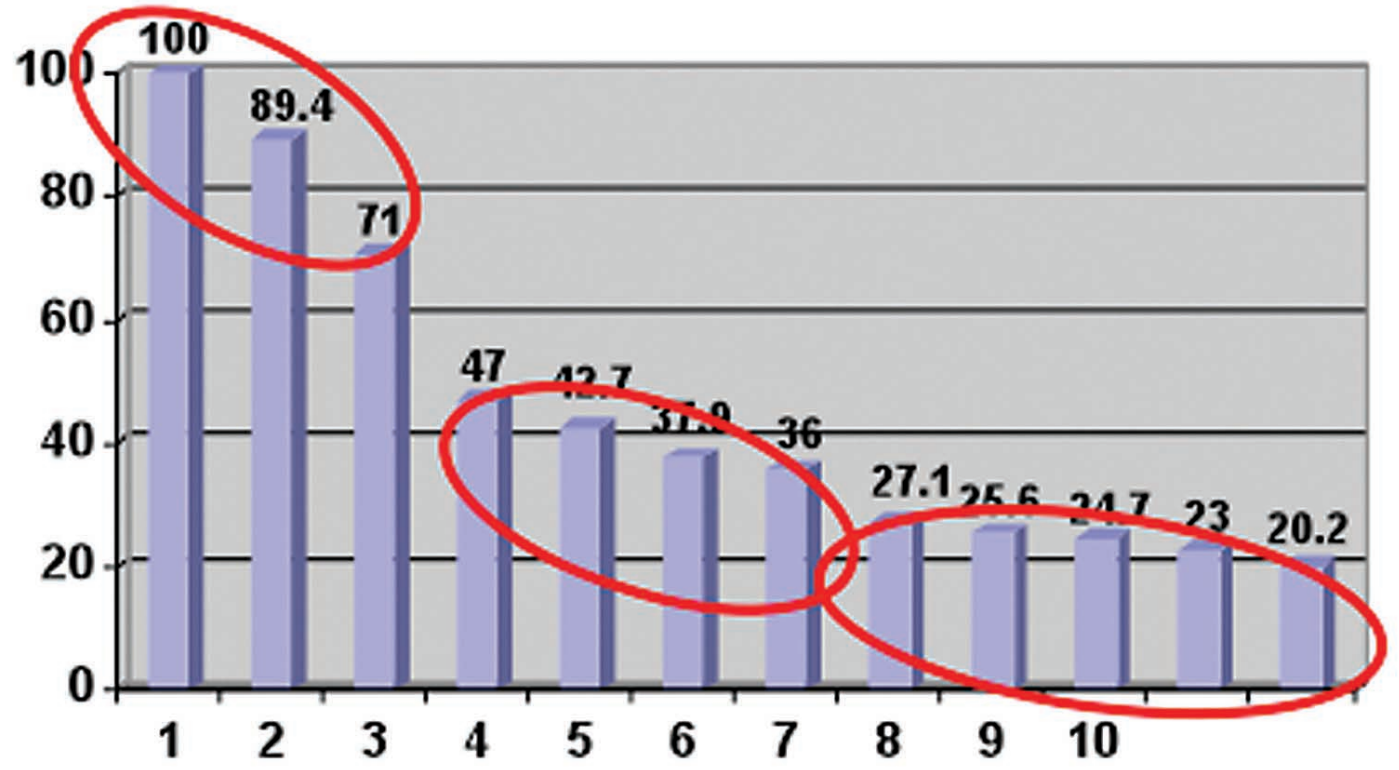

Fig. 3. Representación de los núcleos semánticos de los estudiantes del conjunto SAM. 
Tabla III. Conjunto SAM de los estudiantes sobre el concepto "Buen Profesor Universitario".

\begin{tabular}{clcc}
\hline $\boldsymbol{N}^{\boldsymbol{o}}$ & Palabra Definidora & $\begin{array}{c}\text { Peso Semántico } \\
\text { Valor } \boldsymbol{M}\end{array}$ & $\begin{array}{c}\text { Valor FMG } \\
\text { \% }\end{array}$ \\
\hline 1 & Responsable & 1119 & 100 \\
2 & Respetuoso & 1000 & 89.4 \\
3 & Empático & 794 & 71,0 \\
4 & Inteligente & 526 & 47,0 \\
5 & Puntual & 478 & 42.7 \\
6 & Comprensivo & 424 & 37.9 \\
7 & Motivador & 403 & 36,0 \\
8 & Conocimiento & 303 & 27.1 \\
9 & Amable & 287 & 25.6 \\
10 & Actualizado & 276 & 24.7 \\
11 & Comprometido & 257 & 23,0 \\
$\mathbf{1 2}$ & Exigente & 226 & 20,2 \\
\hline
\end{tabular}

Tabla IV. Palabras definidoras por Carrera sobre el concepto "Buen Profesor Universitario"

\begin{tabular}{|c|c|c|c|c|}
\hline Carrera & $\begin{array}{c}N^{o} \text { palabras } \\
\text { encuesta }\end{array}$ & $\begin{array}{l}N^{o} \text { palabras } \\
\text { definidoras }\end{array}$ & Palabras definidoras & $\%$ \\
\hline Kinesiología & 9 & 135 & $\begin{array}{l}\text { Responsable } \\
\text { Empático } \\
\text { Respetuoso }\end{array}$ & $\begin{array}{r}100 \\
89.4 \\
87.7\end{array}$ \\
\hline Enfermería & 8 & 113 & $\begin{array}{l}\text { Responsable } \\
\text { Respetuoso } \\
\text { Inteligente }\end{array}$ & $\begin{array}{r}100 \\
98,0 \\
45.9\end{array}$ \\
\hline Medicina & 5 & 98 & $\begin{array}{l}\text { Respetuoso } \\
\text { Responsable } \\
\text { Comprometido }\end{array}$ & $\begin{array}{r}100 \\
98.4 \\
71,0\end{array}$ \\
\hline Obstetricia & 9 & 77 & $\begin{array}{l}\text { Empático } \\
\text { Respetuoso } \\
\text { Responsable }\end{array}$ & $\begin{array}{r}100 \\
63.7 \\
45.9\end{array}$ \\
\hline Nutrición & 9 & 107 & $\begin{array}{l}\text { Responsable } \\
\text { Empático } \\
\text { Comprensivo }\end{array}$ & $\begin{array}{r}100 \\
87.1 \\
75.2\end{array}$ \\
\hline Tecnología Médica & 9 & 100 & $\begin{array}{l}\text { Respetuoso } \\
\text { Responsable } \\
\text { Conocimiento }\end{array}$ & $\begin{array}{r}100 \\
63,0 \\
52.1\end{array}$ \\
\hline Odontología & 8 & 162 & $\begin{array}{l}\text { Responsable } \\
\text { Respetuoso } \\
\text { Puntual }\end{array}$ & $\begin{array}{l}100 \\
97.6 \\
94.4\end{array}$ \\
\hline
\end{tabular}

\section{DISCUSIÓN}

Los resultados muestran coincidencia de los conceptos "Responsable" y "Empático", con mayor connotación de un "Buen Profesor Universitario" para docentes y estudiantes, representando para ambos estas características como importantes para el proceso educativo. Surgiendo con ello un significado producto de la experiencia social y cultural propia de estos grupos, como necesarias para la interacción de los mismos, si se considera que todo proceso de enseñanza - aprendizaje es comunicación.
Dentro de las diferencias a destacar entre ambos grupos es el concepto de "Respetuoso", como un actitud personal, el cual aparece con un alto peso semántico para los estudiantes y para el profesorado emerge sólo en el tercer núcleo de palabras. Denotando con ello la relevancia que tiene para el estudiantado el aspecto actitudinal, valórico del docente, relacionado con los aprendizajes fundamentales de Delors del saber ser y saber convivir, recomendados para el educando, que también podrían ser recomendados 
para el profesorado. Otra diferencia que surge es el valor que le dan los docentes al concepto que tienen que ver con el saber sustantivo de una disciplina y a la formación continua, al considerar "Conocimiento", "Estudioso", "Actualizado", "Capacitado" como segundo núcleo semántico de la red, indicando la importancia que le dan al aspecto cognitivo. Aspectos que en la visión de los estudiantes sólo aparecen en el tercer núcleo temático con porcentajes bastantes menores. Es decir, los estudiantes expresan conceptos que dan cuenta de competencias o características valóricas por sobre las actitudes relacionadas con los saberes, a diferencia de los docentes que consideran de mayor valor las competencias relacionadas con los saberes y la actualización de los mismos, por sobre las actitudes y comportamientos.

Cabe hacer notar que estos estudiantes pertenecientes a los últimos cursos de pregrado de las diferentes carreras de la Facultad de Medicina de la Universidad de La Frontera son coincidentes con los obtenidos en los estudiantes de primer año (Cabalín \& Navarro) "Respetuoso" y "Responsable", indicando ello que la permanencia en la Universidad no cambia la idea internalizada de los estudiantes sobre el concepto de "Buen Profesor Universitario". Pudiera ser entonces que estas características sean transversales a todo el proceso de enseñanza - aprendizaje, indistinto de los diferentes niveles educacionales que ello considere (Bá- sica, Media, Universitaria). Frente a los resultados del estudio surge la necesidad de indagar que significa ser responsable y respetuoso para los estudiantes.

Además en los estudiantes emergen los conceptos de "Comprensivo, "Amable", "Motivador" y "Puntual", aspectos que tienen que ver con el trato del docente, conceptos que no aparecen en el grupo de los docentes, quizá porque no lo consideren como importante dentro de las competencias y características de un buen profesor. Estas apreciaciones concuerdan con lo señalado por Cataldi \& Lage donde "los estudiantes solicitan buen trato y que se interesen por ellos", fundamentando sus necesidades principalmente desde un aspecto social.

Cuando hablamos de innovación educativa necesariamente debemos poner el acento en cual será el aporte que esa innovación hará para la mejora del proceso educativo, por lo tanto es necesario que el docente universitario modifique sus prácticas pedagógicas con los estudiantes, que pasa por reconocer el valor y aporte que una entrega de conocimientos junto con una mayor interacción interpersonal o humanización en relación con; respeto, amabilidad, compresión, empatía, entre otros, lo que redundará en una mejora del proceso enseñanza/aprendizaje y es aquí donde el profesor como modelo puede potenciar el saber estar de estos futuros profesionales en la sociedad.

CABALÍN, S. D.; NAVARRO, H. N.; ZAMORA, S. J. \& SAN MARTíN, G. S. Thinking of students and teachers of good university teacher. Faculty of Medicine Universidad de La Frontera. Int. J. Morphol., 28(1):283-290, 2010.

SUMMARY: Currently the quality of a University Professor is heavily influenced by the new roles to be undertaken according to focus educational process on the students. This study aims to explore the representation that students and teachers of the Faculty of Medicine at the Universidad de La Frontera have in relation to the concept "good university professor". The study is based on qualitative research by allowing access to the conceptual world of individuals and networks of meanings using the technique of natural semantic networks. The survey was administered to 104 professors from various careers and 293 senior students. Each of them expressed in ten words their representation of the concept and ranked it according to the importance they assigned. The participants were asked for their consent, assuring anonymity and confidentiality of data. From the instructors emerged 228 words defining the concept, and 248 from the students. Instructors assign more semantic weight to "Responsibility" (100\%), "Empathy" (97.6\%), and "Knowledge" (60.3\%). Students gave priority to "Responsible" (100\%), "Respectful" (89.4\%), and "Empathetic" (71\%) as major attributes of the Good University Professor. On second level instructors include aspects of cognition and knowledge ( $60.3 \%$ knowledge, $49.4 \%$ committed 48.4 updated, $43.3 \%$ studious) and students include aspects of (intelligent 47\%, punctual 42.7\%, commitment 37.9\%, motivating 36\%). The importance that both teachers and students assign to responsibility and empathy (which are related to skills of knowing how to be and knowing how to relate) is highlighted. For professors a second area of importance is given to knowledge's competences, thus giving value to a continuous formation, unlike students for whom this aspect is of no major significance.

KEY WORDS: University professor profile; Semantic networks; Skills.

\section{REFERENCIAS BIBLIOGRÁFICAS}

Arbizu, F. La función docente del profesor universitario. Bilbao, Universidad del País Vasco, 1994.
Ballantine, J. University teaching around the world. Teaching Sociology, 17:291-6, 1989. 
Cabalín, D. \& Navarro, N. Conceptualización de los estudiantes sobre el buen profesor universitario en las carreras de la salud de la Universidad de La Frontera. Int. J. Morphol., 26(4):887-92, 2008.

Clark, S. M. The academia profesión and career: perspectivas and problems. Teaching Sociology, 14:24-34,1986.

Cataldi, Z. \& Lage, F. Un nuevo perfil del profesor universitario. Revista de informática educativa y medios audiovisuales, 1(3):28-33, 2004.

Chomsky, N. Linguagem e pensamento. Río de Janeiro, Ed. Vozes, 1971. p.45.

Corona, C. ¿Qué hace el buen maestro?. La visión del estudiante de ciencias físico matemáticas. Lat. Am. J. Phys. Educ., 2(2):147-151, 2008.

Delors, J. La educación encierra un tesoro. Informe de la UNESCO de la Comisión Internacional sobre la Educación para el Siglo XXI. Barcelona, Grupo Santillana, 1996.

Dewey, J. Democracia y educación. Madrid, Morata, 1995.

Figueroa, J.; González, E. \& Solís, V. Una aproximación al problema del significado: las redes semánticas. Rev. Latinoam. Psicol., 13(3):447-58, 1981.

Knowles, M. Self directed learninig. A guide for learners and Teachers. New York, Association Press, 1975.

Lagunas, I. Las redes semánticas naturales, su conceptualización y su utilización en la construcción de instrumentos. Revista de Psicología Social y Personalidad, 77:8197, 1993.

Manso, J. Reflexiones sobre un nuevo modelo de profesor universitario en Medicina. Una visión crítica de la enseñanza médica. $2^{\circ}$ Congreso Virtual de Cardiología, 2005. Disponible en: http://www.fac.org/scvc/llave/edu/ manso.htm.

Nowakowski, P. Trabajo del profesorado universitario según lo evalúan los estudiantes. Educação, 3(63):54756, 2007.

Santos, M. A. Criterios de referencia sobre calidad del proceso de enseñanza/aprendizaje en la universidad. Primeras Jornadas Nacionales de Didáctica Universitaria, Madrid, Consejo de Universidades, 1990.
Taylor, S. \& Bogdan, R. Introducción a los métodos cualitativos de investigación. La búsqueda de significados. Buenos Aires, Paidós, 1986.

Torres A.; Ruíz J. \& Alvarez, N. La autotransformación del estudiante universitario: más allá de la formación integral. Revista Iberoamericana de Educación, 43(4):1-9, 2007.

Tuning Educational Structures in Europe. Informe Final. Universidad de Deusto - Universidad de Groningen. Edición y Cultura. Sócrates, 2003. pp.81-4.

Valdez, J. L. Las redes semánticas naturales, usos y aplicaciones en psicología social. México, Universidad Autónoma del Estado de México, 1998.

Valdez, J. L. \& Reyes L. Las categorías semánticas y el autoconcepto. Rev. Psicología Social en México, IV:1939, 1992.

Verona, M. La universidad y el profesor universitario: reflexiones y comentarios. Revista de Educación Superior, 33(1):129, 2004.

Dirección para correspondencia:

Prof. Daisy Cabalín Silva

Oficina de Educación Médica

Facultad de Medicina

Universidad de La Frontera

Casilla 54-D

Temuco-CHILE

Email:dcabalin@ufro.cl

Recibido : 09-11-2009

Aceptado: 08-01-2010 